\title{
Fixed Priority Scheduling of Age Constraint Processes
}

\author{
Lars Lundberg \\ Department of Computer Science, University of Karlskrona/Ronneby, \\ S-372 25 Ronneby, Sweden, \\ Lars . Lundbergeide.hk-r.se
}

\begin{abstract}
Real-time systems often consist of a number of independent processes which operate under an age constraint. In such systems, the maximum time from the start process $L_{i}$ in cycle $k$ to the end in cycle $k+1$ must not exceed the age constraint $A_{i}$ for that process. The age constraint can be met by using fixed priority scheduling and periods equal to $A_{i} / 2$. However, this approach restricts the number of process sets which are schedulable.

In this paper, we define a method for obtaining process periods other than $A_{i} / 2$. The periods are calculated in such a way that the age constraints are met. Our approach is better in the sense that a larger number of process sets can be scheduled compared to using periods equal to $A_{\imath} / 2$.
\end{abstract}

\section{Introduction}

Real-time systems often consist of a number of independent periodic processes. These processes may handle external activities by monitoring sensors and then producing proper outputs within certain time intervals. A similar example is a process which continuously monitors certain variables in a database. When these variables or sensors change, the system have to produce certain outputs within certain time intervals. These outputs must be calculated from input values which are fresh, i.e. the age of the input value must not exceed certain time limits. The processes in these kinds of systems operate under the age constraint.

The age constraint defines a limit on the maximum time from the point in time when a new input value appears to the point in time when the appropriate output is produced. Figure 1 shows a scenario where a value $E_{i}$ appears shortly after process $L_{i}$ has started its $k$ :th cycle (denoted $L_{i}^{k}$ ). Process $L_{i}$ starts its execution by reading the sensor or variable. Consequently, $E_{i}$ will not affect the output in cycle $k$. The output $F_{i}$ corresponding to value $E_{i}$ (or fresher) is produced at the end of cycle $k+1$. The age constraint $A_{i}$ is defined as the maximum time between the beginning of the process' execution in cycle $k$ to the end of the process' execution in cycle $k+1$.

A scheduling scheme can be either static or dynamic. In dynamic schemes the priority of a process is decided at run-time, e.g. the earliest deadline algorithm [3]. In static schemes, processes are assigned a fixed priority, e.g. the rate-monotone 
algorithm [4]. Fixed priority scheduling is relatively easy to implement and it requires less overhead than dynamic schemes.

Most studies in this area have looked at scenarios where the computation time and the period of a process are known. However, for age constraint processes the period is not known. We have instead defined a maximum time between the beginning of the process' execution in cycle $k$ to the end of the process' execution in cycle $k+1$. We would like to translate this restriction into a period for the process thus making it possible to use fixed priority schemes.

The age constraint is met by specifying a process period $T_{i}^{\prime}=A_{i} / 2$ (we will use the notation $T_{i}$ for other purposes), thus obtaining a set of processes with known periods $T_{i}^{\prime}$ and computation times $C_{i}$. For such scenarios, it is well known that rate-monotone scheduling is optimal, and a number of schedulability tests have been obtained [4]. Specifying a process period $T_{i}^{\prime}=A_{i} / 2$ for age constraint processes is, however, an unnecessary strong restriction, which do not allow that the start of $L_{i}$ in cycle $k$ and the end in cycle $k+2$ may be separated by a time greater than $3 T_{i}^{\prime}$, whereas the age constraint allows a separation of up to $2 A_{i}=4 T_{i}^{\prime}$.

In this paper we show that, by using rate-monotone scheduling, it is possible to define periods which are better than using periods $T_{i}^{\prime}=A_{i} / 2$. Our method is better in the sense that we will be able to schedule a larger number of process sets than using $T_{i}^{\prime}=A_{i} / 2$.

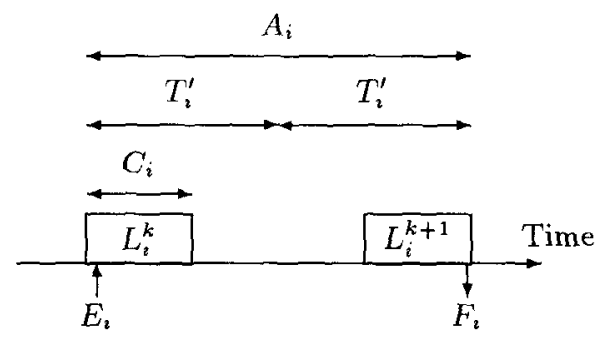

Fig. 1. The age constraint for process $L_{i}$.

\section{Calculating process periods}

Consider a set of $n$ processes $\bar{L}=\left[L_{1}, L_{2}, \ldots, L_{n}\right]$, with associated age constraints $A_{i}$ and computation times $C_{i}$. The priority of each process is defined by its age constraint $A_{i}$. The smaller the value $A_{i}$, the higher the priority of $L_{i}$, i.e. the priority order is the same as for rate-monotone scheduling with $T_{i}^{\prime}=A_{i} / 2$. We assume preemptive scheduling and we order the processes in such a way that $A_{i} \leq A_{i+1}$. 
We start by considering $L_{1}$. This process has the highest priority, and is thus not interrupted by any other process. We want to select as long periods as possible, thus minimizing processor utilization. Obviously, the period of a process must not exceed $A_{i}-C_{i}$. Since $L_{1}$ has the highest priority, it is safe to set the period of $L_{1}$ to $A_{1}-C_{1}$ In order to distinguish our periods from the ones which are simply equal to half the age constraint $A_{i}$, we denote our periods as $T_{i}$, i.e. $T_{1}=A_{1}-C_{1}$.

We now consider process $L_{2}$. The maximum response time $R_{2}$ of process $L_{2}$ is defined as the maximum time from the release of $L_{2}$ in cycle $k$ to the time that $L_{2}$ completes in the same cycle. If the execution of $L_{2}$ is not interrupted by any other process, the response time is simply equal to the computation time $C_{2}$, i.e. $R_{1}=C_{1}$. However, the execution of $L_{2}$ may be interrupted by $L_{1}$. Process $L_{1}$ may in fact interfere with as much as $\left[R_{2} / T_{1}\right\rceil C_{1}$ [3]. Consequently, $R_{2}=C_{2}+\left\lceil R_{2} / T_{1}\right\rceil C_{1}$. The only unknown value in this equation is $R_{2}$. The equation is somewhat difficult to solve due to the ceiling function $\left(\left\lceil R_{2} / T_{1}\right\rceil\right)$. In general there could be many values of $R_{2}$ that solve this equation. The smallest such value represents the worst-case response time for process $L_{2}$. It has been shown that $R_{2}$ can be obtained from this equation by forming a recurrence relationship. The technique for doing this is shown in [3].

The beginning of $L_{2}$ in cycle $k$ and the end of $L_{2}$ in cycle $k+1$ may be separated with as much as $T_{2}+R_{2}$, where $T_{2}$ is the period that we will assign to process $L_{2}$. From the age constraint we know that $T_{2}+R_{2} \leq A_{2}$. In order to minimize processor utilization we would like to select as long a period $T_{2}$ as possible. Consequently, $T_{2}=A_{2}-R_{2}$.

In general, the maximum response time of process $i$ can be obtained from the relation $R_{i}=C_{i}+\sum_{j=1}^{i-1}\left\lceil R_{i} / T_{j}\right\rceil C_{j}$ [3]. When we know $R_{i}$, the cycle time for $L_{i}$ is set to $T_{i}=A_{i}-R_{i}$.

Figure 2 shows a set with three processes $L_{1}, L_{2}$ and $L_{3}$, defined by $A_{1}=8$, $C_{1}=2, A_{2}=10, C_{2}=2, A_{3}=12$ and $C_{3}=2$. From these values we obtain the period $T_{1}=A_{1}-C_{1}=8-2=6$. The maximum response time $R_{2}$ for process $L_{2}$ is obtained from the relation $R_{2}=C_{2}+\left\lceil R_{2} / T_{1}\right\rceil C_{1}=2+\left\lceil R_{2} / 6\right\rceil 2$. The smallest value $R_{2}$ which solves this equation is 4 , i.e. $R_{2}=4$. Consequently, $T_{2}=$ $A_{2}-R_{2}=10-4=6$. The maximum response time $R_{3}$ for process $L_{3}$ is obtained from the relation $R_{3}=C_{3}+\left\{R_{3} / T_{1}\right\rceil C_{1}+\left\lceil R_{3} / T_{2}\right\rceil C_{2}=2+\left\lceil R_{3} / 6\right\rceil 2+\left\lceil R_{3} / 6\right\rceil 2$. The smallest value $R_{3}$ which solves this equation is 6 , i.e. $R_{3}=6$. Consequently, $T_{3}=A_{3}-R_{3}=12-6=6$. In figure 2 , the first release of $L_{1}$ is done at time 2 , the first release of $L_{2}$ is done at time 1 and the first release of $L_{3}$ is done at time 0 .

In the worst-case scenario, process $L_{i}$ may suffer from the maximum response time $R_{i}$ in two consecutive cycles, i.e. in order to meet the age constraint we know that $2 R i \leq A_{i}$. Consequently, there is no use in selecting a $T_{i}$ smaller than $R_{i}$. However, as long as we obtain $T_{i}$ which are longer than or equal to $R_{i}$, the age constraint will be met. Therefore, process $L_{i}$ can be scheduled if and only if $R_{i} \leq A_{i} / 2$. 
$L_{1}$ defined by $A_{1}=8$ and $C_{1}=2$ from this we calculate $T_{1}=6$

$L_{2}$ defined by $A_{2}=10$ and $C_{2}=2$ from this we calculate $T_{2}=6$

$L_{3}$ defined by $A_{3}=12$ and $C_{3}=2$ from this we calculate $T_{3}=6$
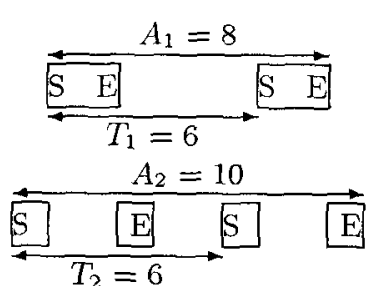

$A_{3}=12$
$E$ denotes the end of a computation

\section{S E}

$S$ denotes the start of a computation

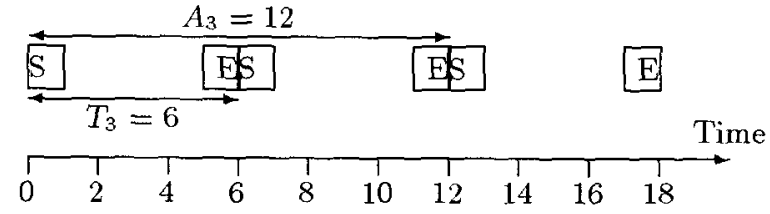

Fig. 2. A set with three processes $L_{1}, L_{2}$ and $L_{3}$, defined by $A_{1}=8, C_{1}=2, A_{2}=$ $10, C_{2}=2, A_{3}=12$ and $C_{3}=2$.

Theorem 1. A set of processes which is schedulable using rate-monotone priority assignment and $T_{i}^{\prime}=A_{i} / 2$ is also schedulable using our scheme.

Proof. The difference between our scheme and rate-monotone with $T_{i}^{\prime}=A_{i} / 2$ is that we use different periods $T_{i}$. Since the process set is schedulable using the $T_{i}^{\prime}$ periods we know that $R_{i}^{\prime} \leq T_{i}^{\prime}(1 \leq i \leq n)$, where $R_{i}^{\prime}$ denotes the maximum response time using the $T_{i}^{\prime}$ periods.

By use of induction, we show that $R_{i} \leq R_{i}^{\prime}(1 \leq i \leq n)$.

- $R_{1}=C_{1} \leq R_{1}^{\prime}=C_{1}$

- If $R_{j} \leq R_{j}^{\prime}(1 \leq j \leq x<n)$, then $T_{j}^{\prime}=A_{j} / 2 \leq A_{j}-R_{j}=T_{j}$. If $T_{j}^{\prime} \leq T_{j}$, then $R_{x+1}=C_{x+1}+\sum_{j=1}^{x}\left\lceil R_{x+1} / T_{j}\right\rceil C_{j} \leq R_{x+1}^{\prime}=C_{x+1}+\sum_{j=1}^{x}\left\lceil R_{x+1}^{\prime} / T_{j}^{\prime}\right\rceil C_{j}$.

Consequently, $R_{i} \leq R_{i}^{\prime} \leq T_{i}^{\prime}=A_{i} / 2$, i.e. $R_{i} \leq A_{i} / 2$, which means that process $L_{i}(1 \leq i \leq n)$ can be scheduled using our scheme.

Consider the processes in figure 2. If we would have used the periods $A_{i} / 2$, we would have got $T_{1}^{\prime}=4, T_{2}^{\prime}=5$ and $T_{3}^{\prime}=6$. This would have resulted in a utilization of $C_{1} / T_{1}^{\prime}+C_{2} / T_{2}^{\prime}+C_{3} / T_{3}^{\prime}=2 / 4+2 / 5+2 / 6=1.23>1$, i.e. the process set would not have been schedulable. Consequently, our scheme is better than rate-monotone and $T_{i}^{\prime}=A_{i} / 2$ in the sense that we are able to schedule a larger number of process sets.

\section{Simple analysis}

In the scheme that we propose, the period of a process depends on the priority of the process. The period for a process $L_{i}$ gets shorter if the priority of $L_{i}$ is reduced and vice versa. This property makes our scheme hard to analyze. However, for the limited case when there are two processes, a thorough analysis is possible. 
Theorem 2. The priority assignment used in our scheme is optimal for all sets containing two processes.

Proof. Consider two processes $L_{1}$ and $L_{2}$, such that $A_{1} \leq A_{2}$. Assume that these two processes are schedulable if the priority of $L_{2}$ is higher than the priority of $L_{1}$. We will now show that if this is the case, the two processes are also schedulable if $L_{1}$ has higher priority than $L_{2}$.

If $L_{1}$ and $L_{2}$ are schedulable when the priority of $L_{2}$ is higher than the priority of $L_{1}$, then $R_{1}=C_{1}+\left\lceil R_{1} /\left(A_{2}-C_{2}\right)\right\rceil C_{2}=C_{1}+k C_{2} \leq A_{1} / 2$ (for some integer $k>0$ ). Consequently, $C_{2} \leq A_{1} / 2-C_{1}$.

If we consider the opposite priority assignment we know that the schedulability criterion is that $R_{2}=C_{2}+\left[R_{2} /\left(A_{1}-C_{1}\right)\right] C_{1} \leq A_{2} / 2$. Since $C_{2} \leq A_{1} / 2-C_{1}$, we know that the maximum interference from process $L_{1}$ on process $L_{2}$ is $C_{1}$, i.e. $R_{2}=C_{2}+C_{1}$. Since $C_{2} \leq A_{1} / 2-C_{1}$ and $R_{2}=C_{2}+C_{1}$, we know that $R_{2} \leq A_{1} / 2$, and since $A_{1} \leq A_{2}$, we know that $R_{2} \leq A_{2} / 2$, thus proving the theorem.

Theorem 3. All sets containing two processes $L_{1}$ and $L_{2}$ for which $C_{1} /\left(A_{1}-\right.$ $\left.C_{1}\right)+C_{2} /\left(A_{2}-C_{2}\right)$ is less than $2(\sqrt{2}-1)=0.83$ are schedulable using our scheme, and there are process sets containing two processes for which $C_{1} /\left(A_{1}\right.$ $\left.C_{1}\right)+C_{2} /\left(A_{2}-C_{2}\right)=2(\sqrt{2}-1)+e$ (for any $\left.e>0\right)$ which are not schedulable using our scheme.

Proof. We assume that $A_{1} \leq A_{2}$, and that we use our scheme for calculating periods and priorities.

We want to find the minimum value $C_{1} /\left(A_{1}-C_{1}\right)+C_{2} /\left(A_{2}-C_{2}\right)$, such that the process set is not schedulable. We know that as long as $C_{1} /\left(A_{1}-C_{1}\right) \leq 1$, process $L_{1}$ can be scheduled. This is a trivial observation.

Process $L_{2}$ can be scheduled if $R_{2} \leq A_{2} / 2$, i.e. we want to minimize $C_{1} /\left(A_{1}-\right.$ $\left.C_{1}\right)+C_{2} /\left(A_{2}-C_{2}\right)$ under the constraint that $R_{2}=C_{2}+\left\lceil R_{2} /\left(A_{1}-C_{1}\right)\right\rceil C_{1}>$ $A_{2} / 2$.

If $\left[R_{2} /\left(A_{1}-C_{1}\right)\right]=k$ (for some integer $k>0$ ), then the minimum for $C_{1} /\left(A_{1}-C_{1}\right)+C_{2} /\left(A_{2}-C_{2}\right)$ is obtained when $R_{2}=k\left(A_{1}-C_{1}\right)=C_{2}+\left\lceil R_{2} /\left(A_{1}-\right.\right.$ $\left.\left.C_{1}\right)\right\rceil C_{1}=C_{2}+k C_{1}=>C_{2}=k\left(A_{1}-2 C_{1}\right)$. Consequently, we want to find the $k$ which minimizes $C_{1} /\left(A_{1}-C_{1}\right)+k\left(A_{1}-2 C_{1}\right) /\left(A_{2}-k\left(A_{1}-2 C_{1}\right)\right)$. Since, $C_{2}=$ $k\left(A_{1}-2 C_{1}\right) \leq A_{2} / 2$ we see that $A_{2}-k\left(A_{1}-2 C_{1}\right)>0$, and since $0 \leq A_{1}-2 C_{1}$, we see that the minimum for $C_{1} /\left(A_{1}-C_{1}\right)+k\left(A_{1}-2 C_{1}\right) /\left(A_{2}-k\left(A_{1}-2 C_{1}\right)\right)$ is obtained for $k=1$. Consequently, we want to minimize $C_{1} /\left(A_{1}-C_{1}\right)+\left(A_{1}\right.$ $\left.2 C_{1}\right) /\left(A_{2}-\left(A_{1}-2 C_{1}\right)\right)$, under the constraint that $R_{2}=A_{1}-C_{1}>A_{2} / 2$. The minimum is obviously obtained when $A_{1}-C_{1}$ is as small as possible, i.e. when $A_{1}-C_{1}=A_{2} / 2+e$ (for some infinitely small positive number $e$ ). Consequently, we want to minimize $C_{1} /\left(A_{2} / 2+e\right)+\left(A_{2} / 2+e-C_{1}\right) /\left(A_{2}-\left(A_{2} / 2+e-C_{1}\right)\right)$. Without loss of generality we assume that $A_{2}=2$. In that case we obtain the following function (disregarding $e$ )

$$
f\left(C_{1}\right)=C_{1}+\left(1-C_{1}\right) /\left(1+C_{1}\right)
$$


From this we obtain the derivative of $f$ :

$$
f^{\prime}\left(C_{1}\right)=1+\left(\left(1-C_{1}\right)-\left(1+C_{1}\right)\right) /\left(1+C_{1}\right)^{2}
$$

By setting $f^{\prime}\left(C_{1}\right)=0$ we will find the values $C_{1}$ which minimizes $f$.

$$
1+\left(\left(1-C_{1}\right)-\left(1+C_{1}\right)\right) /\left(1+C_{1}\right)^{2}=0 \Rightarrow>1+2 C_{1}+C_{1}^{2}+1-2 C_{1}=0=>C_{1}=\sqrt{2}-1
$$

From this we that $\min f\left(C_{1}\right)=f(\sqrt{2}-1)=2(\sqrt{2}-1)=0.83$.

It is interesting to note that the schedulability bound for $C_{1} /\left(A_{1}-C_{1}\right)+$ $C_{2} /\left(A_{2}-C_{2}\right)$ is the same as the schedulability bound for rate-monotone scheduling and two processors. However, in that case we have $C_{1} / T_{1}^{\prime}+C_{2} / T_{2}^{\prime}=2 C_{1} / A_{1}+$ $2 C_{2} / A_{2}=0.83$. At this point we do not know if it is a coincident that the values are the same or not. It would be interesting to examine the case with three processes and see if the schedulability bound for $C_{1} /\left(A_{1}-C_{1}\right)+C_{2} /\left(A_{2}-\right.$ $\left.C_{2}\right)+C_{3} /\left(A_{3}-C_{3}\right)=3(\sqrt[3]{2}-1)=0.78$, which is the bound for rate-monotone scheduling and three processes.

\section{Improving the scheme}

In the previous sections we assumed that the interference from a higher priority process $L_{j}$ affected the maximum response time $R_{j+x}$ for a process $L_{j+x}$ according to the formula $R_{j+x}=C_{j+x}+\cdots+\left\lceil R_{j+x} / T_{j}\right\rceil C_{j}$. However, if $T_{j+x}=k T_{j}$ (for some integer $k>0$ ), we can adjust the phasing of $L_{j}$ and $L_{j+x}$ in such a way that the interference of $L_{j}$ on $L_{j+x}$ is limited to $\left(\left\lceil R_{j+x} / T_{j}\right\rceil-1\right) C_{j}$ (see figure 3 ). The phasing is adjusted in such a way that a release of process $L_{j+x}$ always occurs at exactly the same time as a release of process $L_{j}$. Consequently, if $T_{j+x}<k T_{j} \leq T_{j+x}-C_{j}$ we can extend the period of $L_{j+x}$ to $k T_{j}$. In order to distinguish the periods obtained when using the optimized version of the scheme from the ones obtained using the unoptimized version, we denote the optimized period for process $L_{j}$ as $t_{j}$.

In order to obtain the optimized periods $t_{j}$, for a set containing $n$ processes, we start with the periods $T_{j}$ and we then use the following algorithm:

$$
\begin{aligned}
& t_{1}=T_{1} \\
& \text { for } y=2 \text { to } n \text { loop } \\
& t_{y}=T_{y} \\
& \text { for } j=1 \text { to } y-1 \text { loop } \\
& \text { if } t_{y}<k t_{j} \leq T_{y}-C_{j} \text { then } t_{y}=k t_{j} \\
& \text { end loop } \\
& y=y+1
\end{aligned}
$$

The execution of the process set is started by releasing all processes at the same time. 


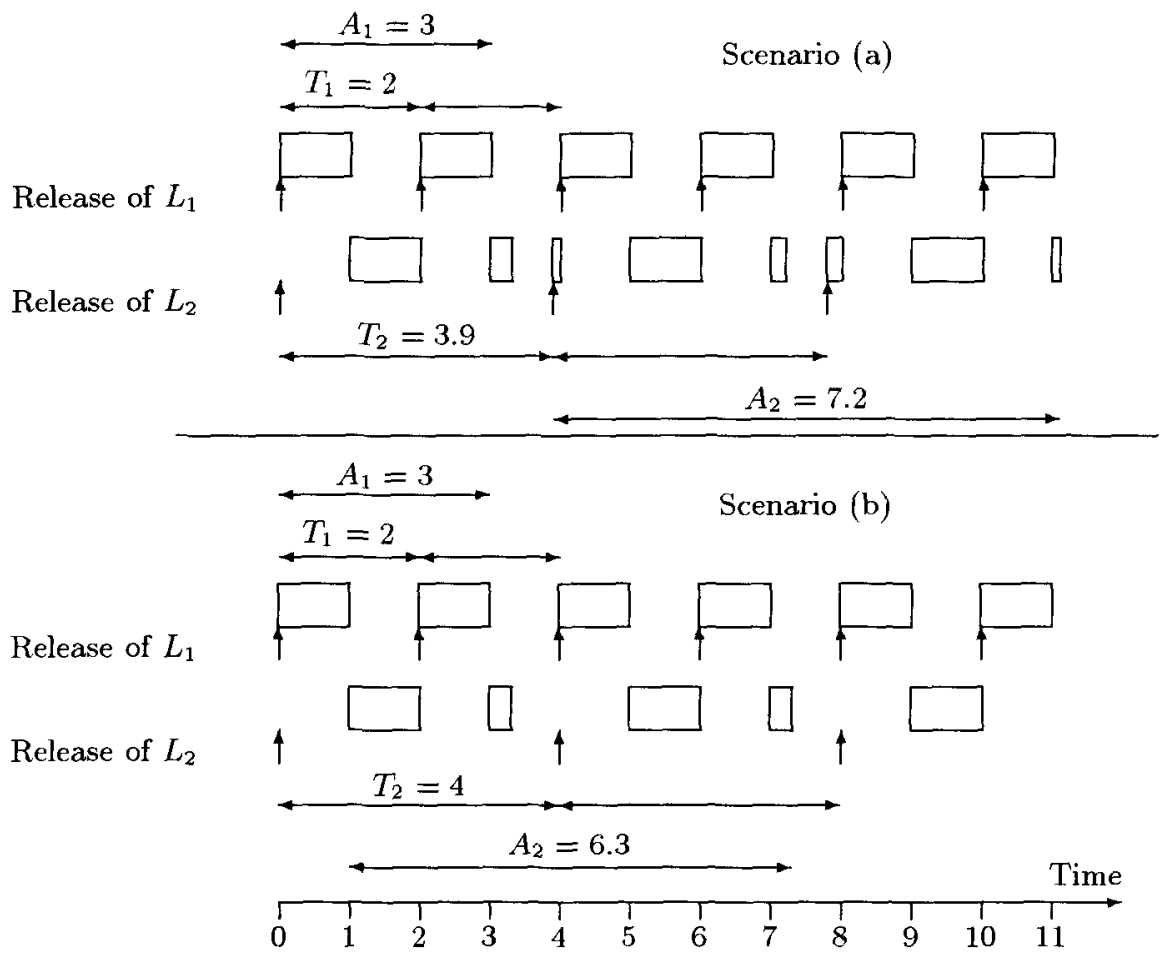

Fig. 3. In scenario (a) there are two processes $L_{1}$ and $L_{2}$. Process $L_{1}$ has a period $T_{1}=2$ and a computation time $C_{1}=1$. Process $L_{2}$ has a period $T_{2}=3.9$ and a computation time $C_{2}=1.3$. In this scenario we are able to meet the age constraints $A_{1}=3$ and $A_{2}=7.2$, i.e. $A_{2}=T_{2}+R_{2}=T_{2}+C_{2}+2 C_{1}=3.9+1.3+2=7.2$. In scenario (b) we consider the same processes, with the exception that the period of $L_{2}$ has been extended, i.e. $T_{2}=2 T_{1}=4$. The phasing of $L_{1}$ and $L_{2}$ has also been adjusted such that a release of $L_{2}$ always coincides with a release of $L_{1}$. In this scenario we are able to meet the age constraints $A_{1}=3$ and $A_{2}=6.3$, i.e. $A_{2}=T_{2}+R_{2}=T_{2}+C_{2}+C_{1}=4+1.3+1=6.3$. Consequently, by extending the period of $L_{2}$ we were able to meet tougher age constraints. 
Theorem 4. A set of processes which is schedulable using the unoptimized periods $T_{i}$ and an arbitrary phasing of processes is also schedulable using the optimized periods $t_{i}$, provided that we are able to adjust the phasing of the processes.

Proof. Let $r_{i}$ denote the maximum response time for process $L_{i}$ using the periods $t_{i}$ and the optimized phasing. Obviously, $T_{i} \leq t_{i}$. From this we conclude that $r_{i}=C_{i}+\sum_{j=1}^{i-1}\left\lceil r_{i} / t_{j}\right\rceil C_{j} \leq R_{i}=C_{i}+\sum_{j=1}^{i-1}\left\lceil R_{i} / T_{j}\right\rceil C_{j}$. Consequently, if $R_{i} \leq$ $A_{i} / 2$, then $r_{i} \leq A_{i} / 2$.

Figure 4 shows a process set which is schedulable using the improved version of our scheme. This process set would not have been schedulable using the unoptimized version of our scheme. Consequently, the improved version of the scheme is better in the sense that we are able to schedule a larger number of process sets.

$L_{1}$ defined by $A_{1}=7$ and $C_{1}=1$ from this we calculate $t_{1}=6$

$L_{2}$ defined by $A_{2}=8$ and $C_{2}=2$ from this we calculate $t_{2}=6$
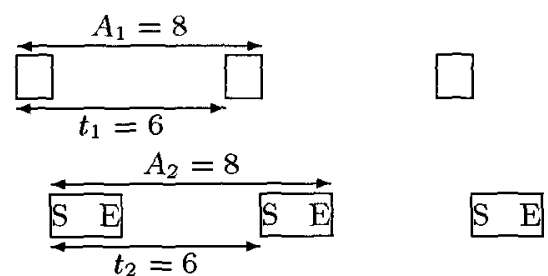

$L_{3}$ defined by $A_{3}=9$ and $C_{3}=3$ from this we calculate $t_{3}=6$

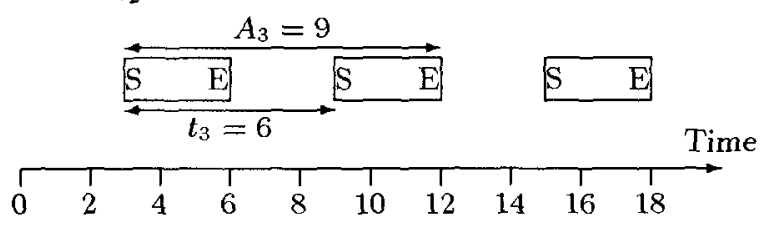

Fig. 4. Three processes which are schedulable using the improved scheme.

\section{Conclusions}

In this paper a method for scheduling age constraint processes has been presented. An age constraint process is defined by two values: the maximum time between the beginning of the process' execution in cycle $k$ to the end of the process' execution in cycle $k+1$ (the age constraint), and the maximum computation time in each cycle, i.e. the period of each process is not explicitly defined. We present an algorithm for calculating process periods. Once the periods have been calculated the process set can be executed using preemption and fixed priority scheduling. The priorities are defined by the rate-monotone algorithm.

Trivially, the age constraint can be met by using periods equal to half the age constraint. However, the periods obtained from our method are better in the sense that they make it possible to schedule a larger number of process sets 
than we would have been able to do if we had used periods equal to half the age constraint. All process sets which are schedulable using periods equal to half the age constraint are also schedulable using our method.

A simple analysis of our method shows that the rate-monotone priority assignment algorithm is optimal for all process sets containing two processes, using our process periods. We also show that all process sets with two processes, for which $C_{1} /\left(A_{1}-C_{1}\right)+C_{2} /\left(A_{2}-C_{2}\right)$ is less than $2(\sqrt{2}-1)=0.83$, are schedulable using our scheme. There are, however, process sets containing two processes for which $C_{1} /\left(A_{1}-C_{1}\right)+C_{2} /\left(A_{2}-C_{2}\right)=2(\sqrt{2}-1)+e($ for any $e>0)$ which are not schedulable using our scheme, i.e. we provide a simple schedulability test for process sets containing two processes.

We also define an improved version of our method. The improved version capitalizes on the fact that there is room for optimization when the period of one process is an integer multiple of the period of another process. The improved version of the method is better than the original version in the sense that we are able to schedule a larger number of process sets. All process sets which are schedulable using the original version are also schedulable using the improved version.

Previous work on age constraint process have concentrated on creating cyclic interleavings of processes [1]. Other studies have looked at age constraint proesses which communicate [5]. One such scenario is scheduling of age constraint ocesses in the context of hard real-time database systems [2].

\section{References}

1. W. Albrecht, and R. Wisser, "Schedulers for Age Constraint Tasks and Their Performance Evaluation", In Proceedings of the Third International Euro-Par Conference, Passau, Germany, August 1997.

2. N. C. Audsley, A. Burns, M.F. Richardson, and A.J. Wellings, "Absolute and Relative Temporal Constraints in Hard Real-Time Databases", In Proceedings of IEEE Euromicro Workshop on Real-Time Systems, Los Alamitos, California, February 1992.

3. A. Burns, and A. Wellings, "Real-Time Systems and Programming Languages", Addison-Wesley, 1996.

4. J.P. Lehoczky, L. Sha, and Y. Ding, "The rate monotone scheduling algorithm: exact characterization and average case behavior", In Proceedings of IEEE 10th Real-Time Systems Symposium, December 1989.

5. X. Song, and J. Liu, "How Well can Data Temporal Consistency be Maintained", In Proceedings of the 1992 IEEE Symposium on Computer Aided Control Systems design, Napa, California, USA, March 1992. 